\title{
Osteitis Condensans Ilii: Case Report
}

\author{
Berat Meryem Alkan*, Hatice Karaarslan, Esra Eroğlu, Ayşenur Alemdar, Saygın Yamçiçi, \\ Özge Ardıçoğlu
}

PMR Clinic, Atatürk Education and Research Hospital, Ankara, Turkey.

Email: berat.alkan@ttmail.com

Received July $8^{\text {th }}, 2011$; revised July $31^{\text {st }}, 2011$; accepted August $8^{\text {th }}, 2011$.

\begin{abstract}
Back pain is one of the most frequent complaints during the last period of pregnancy. In a small group of patients the persistent pain during the postpartum period may be secondary to osteitis condensans ilii (OCI), and rarely can occur suddenly in the elderly. OCI is a rare benign pathology, low back or buttock pain should be suspected in patients coming from. Although the exact etiology is unknown, it is suggested that the increased mechanical stress across the sacroiliac joint, depending on the mechanical stress, changes may develop in the iliac bone. In practice, it is important to recognise this disease as it can be potentially confused with similar causes of axial low back pain such as seronegative spondyloarthropathy, metastatic disease, or sacroilitis. Here two woman patients with low back pain and buttock pain secondary to unilateral an uncommon benign condition called osteitis condensans ilii is presented. This report emphasis the salient features and the differential diagnosis in diagnosing and managing this rare pathology.
\end{abstract}

Keywords: Osteitis Condensans Ilii, Low Back Pain, Triangular Sclerosis

\section{Introduction}

Osteitis condensans ilii (OCI) is an uncommon incidental finding on plain X-ray, characterized by sclerosis of predominantly the iliac bone adjacent to an otherwise normal sacroiliac joint. First described in 1926, OCI is seen almost exclusively in women. It is usually bilateral and symmetrical. Early reports suggested pregnancy and trauma as causative mechanisms [1]. Although It is generally regarded as a self-limiting phenomenon and is mostly asymptomatic, in a small group of patients may be develop the persistent pain secondary to osteitis condensans ilii (OCI). This case report highlights the salient features and discusses the differential diagnosis and management of this benign condition.

\section{Case Report}

A 45 year old Turkish woman presented with low back pain and left buttock pain. The symptoms started during the postpartum period of her pregnancy. The pain was gradual in onset and then became constant. She described the pain as being across her lower back and left buttock and it occasionally radiated down the back of her left leg but not beyond the knee. Coughing or sneezing did not aggravate the pain but she generally experienced discomfort in most activities, particularly if she crouched or bent down. She had never complained of any numbness or "pins and needles" nor weakness in her legs. She had no history suggestive of morning stiffness or other joint involvement She had no family history of joint disorders. She is a dance teacher. Clinically, her body mass index was 28 . She had a slightly increased lumbar lordosis and her gait was normal. She had no wasting of muscles in the lower extremities. She was able to do a tiptoe and heel walking without any difficulty. Flexion, extension, rotation, and lateral bending of her lumbar spine were normal. On palpation, she had no specific bony or paraspinal muscle tenderness. She could demonstrate a straight leg raise up to 90 degrees on both sides and sciatic stretch test, femoral stretch test, Faber and Mennel tests were negative bilaterally. Power, tone, reflexes, and sensation were normal bilaterally. Chest expansion and schober test were normal. Her baseline blood investigations, which were all normal. An anteroposterior plane radiograph of her lumbar spine is shown in Figure 1.

The anteroposterior plane radiograph of the lumbar spine shows unilateral triangular shaped sclerosis around the lower part of the left sacroiliac joint. The sclerosis is mainly affecting the iliac side of the left sacroiliac joint. There is no evidence of narrowing or irregularity in the joint space on both sides. These features are diagnostic of OCI, which is a benign self limiting condition. It is important to recognise this entity as it can be potentially 
confused with other disorders, as mentioned below in the discussion.

The other patient with right hip pain, 56 years old, she maintains an active lifestyle after the retirement. During and after pregnancy has never been such a complaint. Pain continues for a month and could not sleep the pain was learned in the last 24 hours. She could not walk because of pain. Clinically, her body mass index was 32 . She had no wasting of muscles in the lower extremities. She was able to do a tiptoe and heel walking without any difficulty. Flexion, extension, rotation, and lateral bending of her lumbar spine were normal.

Faber test positive on the right. Power, tone, reflexes, and sensation were normal bilaterally. Chest expansion and schober test were normal. Her baseline blood investtigations, which were all normal. Taken lumbosacral radiography (Figure 2) and X-ray tomography (Figure 3) of the pelvis, right iliac captured areas had the typical triangular sclerosis. Treatment in OCI is conservative with physiotherapy and analgesia as required. Our pa- tients had an anti-inflammatory drugs and made a satisfactory recovery.

\section{Discussion}

Low back pain (LBP) is a common and often disabling condition that deserves attention [2]. Back pain is one of the most frequent complaints during the last period of pregnancy, and high incidence has been described in several studies $[3,4]$. Many authors believe that about half of pregnant women suffer from it [5]. The mechanism behind back pain during pregnancy is not clear. Pregnancy increases the hormonal influence on joints and tendons. Together with an increased biomechanical strain on ligaments, muscles, and the skeleton, this is probably the most common cause of back pain during pregnancy [6]. In a small group of patients the persistent pain during the postpartum period may be secondary to OCI.

OCI is a benign condition typically seen after pregnancy and is not associated with any inflammatory

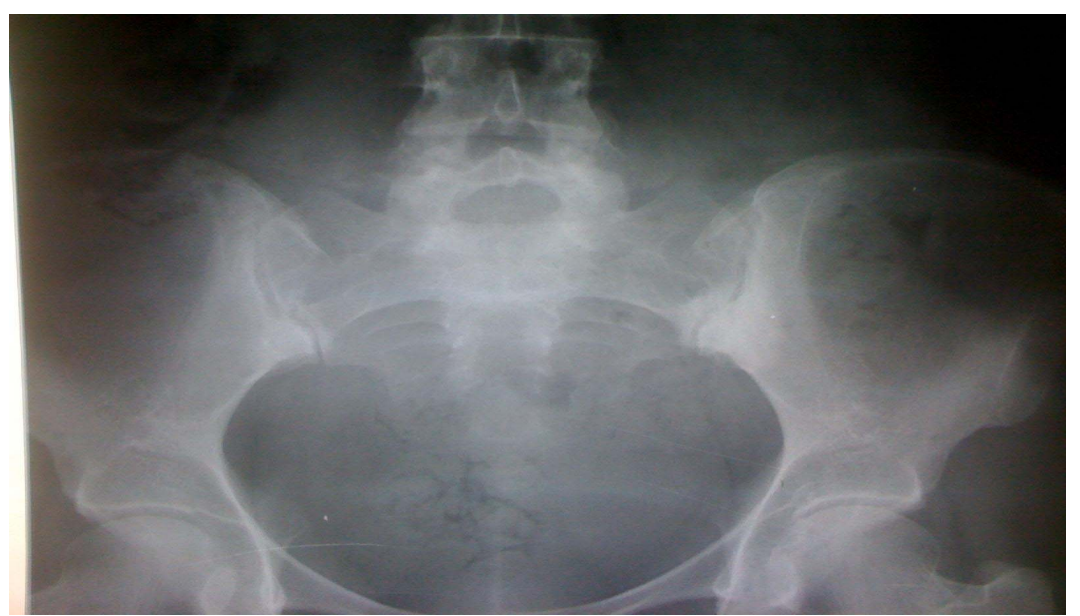

Figure 1. Anteroposterior plain radiograph of lumbar spine: Triangular shaped sclerosis around the lower part of the left sacroiliac joint.

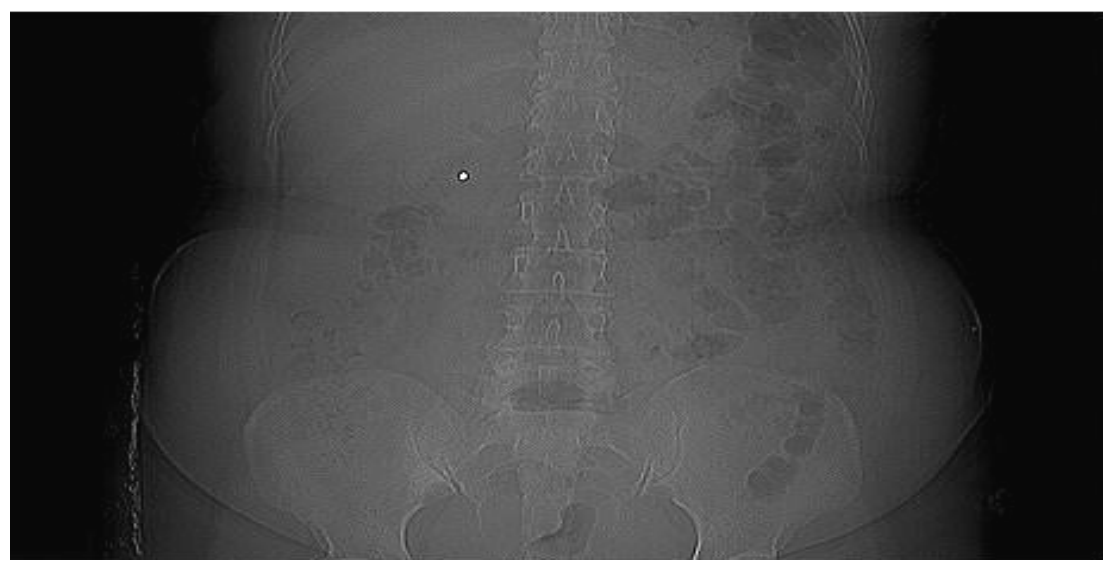

Figure2. Lumbosacral radiography: Right iliac captured areas had the typical triangular sclerosis. 


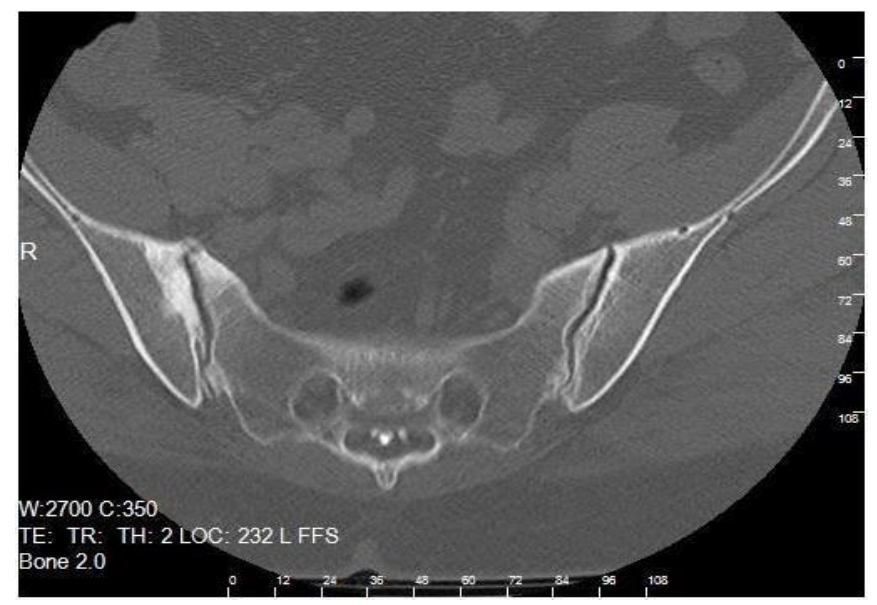

Figure 3. X-ray tomography of the pelvis: Rright iliac captured areas had the typical triangular sclerosis.

arthritis. 56 The prevalence of OCI in the general population has been estimated to be between 0.9 and $2.5 \%$ [7]. It is essentially a radiological diagnosis. The iliac bone adjacent to the sacroiliac joint is predominantly affected and usually, although not invariably, it is a bilateral and relatively symmetric process. It is more common in women of childbearing age group. Rarely, nulliparous women and men may be affected and it can be unilateral. The radiological appearance in $\mathrm{OCI}$ is characteristic with well defined triangular sclerosis on the iliac aspect of the sacroiliac joint. The bony eburnation involves the inferior portion of the bone and the apex of the sclerosis can extend up to the auricular portion of the ilium. In $\mathrm{OCI}$, the joint space is preserved and the articular margins are intact, unlike in other forms of sacroiliitis [8]. The aetiology of OCI is not clear and many theories have been postulated. The most common theory seems to be that mechanical strain of the sacroiliac joint leads to premature joint degeneration and arthritis [9]. Patients afflicted with OCI usually complain of intermittent axial low back pain, which may radiate into the buttocks and posterior thighs in a non-radicular fashion .Patients do not generally complain of systemic complaints (e.g., weight loss, malaise, and chills) [7]. Pregnant patients may complain of axial low back pain in the final trimester or immediately after delivery; the pain may be recurrent with subsequent pregnancies [10]. On physical examination the patients may have a positive FABER test and focal tenderness over the sacroiliac joint. Patients may have increased lumbar lordosis, moderate obesity, and lumbar extensor muscle spasm. Patients with OCI do not have any motor or sensory deficits. Active root tension signs (e.g., straight leg raise test) are negative, and there is no evidence of myelopathy. Interestingly, tests for sacroiliac disease have also been found to be negative [10].

The radiological features and the clinical history are diagnostic of OCI, but other differential diagnoses include seronegative spondyloarthropathies, renal osteodystrophy, lymphoma, Paget's disease, and primary hyperparathyroidism. Normal blood parameters and seronegativity exclude the majority of these disorders. Polyarthralgia in peripheral joints have been noted in a small group of patients with OCI, although significant inflammatory articular findings are generally absent [7]. Unlike OCI, ankylosing spondylitis AS is associated with bony erosions and joint space narrowing (greater then $2 \mathrm{~mm}$ ) on radiographic images [11]. OCI is also generally not associated with the presence of HLA B27 antigens which are found in AS.

To conclude: OCI is a benign self limiting condition seen in women with low back pain. It is important to recognise this entity as it may potentially be confused with sacroiliitis from other disorders like ankylosing spondylitis and inflammatory arthritis. It is more common in women of childbearing age group. Rarely, nulliparous women, elderly and men may be affected and it can be unilateral. Conservative treatment with analgesics and physiotherapy are the mainstay of management in OCI.

\section{REFERENCES}

[1] K. Jenks, G. Meikle, A. Gray and S. Stebbings, "Osteitis Condensans Ilii: A Significant Association with Sacroiliac Joint Tenderness in Women," International Journal of Rheumatic Diseases, Vol. 12, No. 1, 2009, pp. 39-43. doi:10.1111/j.1756-185X.2009.01378.x

[2] E. Berker, "Epidemiology and Risk Factors for Low Back Pain," Turkish Journal of Physical Medicine and Rehabilitation, Vol. 5, No. 44, 1998, pp. 8-12.

[3] J. D. Heckman and R. Sassard, "Muscoloskeletal Considerations in Pregnancy," Journal of Bone and Joint Surgery, Vol. 76, No. 11, 1994, pp. 1720-1730.

[4] P. Kristiansson, K. Svärdsudd and B. von Schoultz, 
"Back Pain during Pregnancy," Spine, Vol. 21, No. 6, 1996, pp. 702-709.

doi:10.1097/00007632-199603150-00008

[5] M. M. LaBan, S. Viola, D. A. Williams and A. M. Wang, "Magnetic Resonance İmaging of the Lumbar Herniated Disc in Pregnancy," American Journal of Physical Medicine \& Rehabilitation, Vol. 74, No. 1, 1995, pp. 5961. doi:10.1097/00002060-199501000-00010

[6] G. Berk, M. Hammar, J. M. Nielsen, U. Linden and J. Thorblad, "Low Back Pain during Pregnancy," Obstetrics \& Gynecology, Vol. 71, No. 1, 1988, pp. 71-75.

[7] R. Mitra, "Osteitis Kondensans Ilii," Rheumatology International, Vol. 30, No. 3, 2010, pp. 293-296. doi:10.1007/s00296-009-1100-7

[8] R. Vadivelu, T. P. Gren and R. Bhatt, "An Uncommon
Cause of Back Pain in Pregnancy," Journal of Postgraduate Medicine, Vol. 81, No. 951, 2005, pp. 65-67. doi:10.1136/pgmj.2003.015370

[9] F. L. Shipp, G. E. Haggart, "Further Experience in the Management of Osteitis Condensans Ilii," Journal of Bone and Joint Surgery, Vol. 32, No. 4, 1950, pp. 841847.

[10] F. L. Shipp and G. E. Haggart, "Further Experience in the Management of Osteitis Condensans Ilii," Journal of Bone and Joint Surgery, Vol. 32, 1950, pp. 841-847.

[11] I. Olivieri, G. Gemignani, E. Camerini, et al., "Differential Diagnosis between Osteitis Condensans İlii and Sacroiliitis," The Journal of Rheumatology, Vol. 17, No. 11, 1990, pp. 1504-1512. 